\title{
An Approach to Extend Electron Holography into Characterization on Dopant Profiles for FinFET of Nanometer Semiconductor Device in Wafer-foundries
}

\author{
Wayne Zhao and Travis Mitchell \\ GLOBALFOUNDRIES US Inc., Malta, New York, United States
}

One theme of efforts in wafer-foundries is to accommodate clients' need in constantly driving for miniaturization of semiconductor device with nanometer transistors as the basic building block [1 2]. To synchronize with the device shrinkage, many integration schemes and novel engineering techniques target to enhance carrier mobility are developed. An in-depth understanding of dopant profiles is one of these critical steps to facilitate device development and engineering for product integration and yield enhancement. However, due to extremely low concentrations of these dopant profiles, which are usually with a concentration in a range of $10^{+20}$ atoms per cubic centimeter, coupled with extremely small dimensions of these implantation zones, it is extremely challenging even for the Analytical Transmission Electron Microscopy (Analytical TEM or AEM) techniques with X-ray Energy Dispersive Spectroscopy (XEDS) and Electron Energy Loss Spectroscopy (EELS) to delineate effectively. As displayed in Fig-1a, XEDS mapping clearly delineated the n-FET enhancement zone of SiP. However, restricted by the detection limit, XEDS (even EELS) is unable to effectively differentiate the dopant wells underneath. Therefore, more alternative technical approaches that are more sensitive to dopant profiles have to be explored. electron holography is one of these approaches that addresses this challenge [2]. Electron holography is sensitive to TEM-sample thickness, which typically requires a TEM lamellar thickness of 200 300nm, ideally. This DEFINITELY hinders the straightforward application to characterize FinFET device, with Fin width (edge-on view) down to $14 \mathrm{~nm}$ and below. In this article, we report one of our experiments that possibly shed a light to extend electron holography into characterization of dopant profiles for 14nm FinFET, as shown in Figs-1b 1e. The examples demonstrated here are comparison of dopant profiles in short and long gate channel effects. Specifically, TEM foils were extracted from devices with fins longer than 400nm longitudinally, even if the fin width itself (from the edge-on cross-sectional view) was still 14nm! The electron holograms were acquired by a Lorentz lens in an ultrahigh resolution Analytical TEM with an Image Corrector, specifically, a Titan model manufactured by Thermal Fisher Scientific (TFS), operating at an acceleration voltage of $200 \mathrm{KV}$. Then, delta in inner potential, between the p-n junction across dopant wells, and dopant well against dopant free zone in pure Si, were postprocessed and extracted by HoloWorks5 software package, as shown in Figs-1b 1e. To fully understand the integrity of the dopant zones, TEM foils were machined along the Fin / perpendicular to Gate, and along the Gate / perpendicular to Fins, respectively, with corresponding electron holograms displayed in Figs-1b and 1d. Electron holography parameters achieved, at a biprism Bias voltage 150V, from these TEM samples, on the Cs image corrected Titan at Fab 8 include: (1) fringe spacing: $2.7 \mathrm{~nm}$ for Fig-1b, and $2.2 \mathrm{~nm}$ for Fig-1d; (2) fringe contrast: 15\% for Fig-1b, and 18\% for Fig-1d; and (3) inner potential delta was $\sim 0.4 \mathrm{~V}$ across p-/n-junctions for along the Fin case in Fig-1b, and $\sim 0.25 \mathrm{~V}$ (dopant well vs. dopant-free pure $\mathrm{Si}$ ) for along the Gate case in Fig-1d, respectively. For the case in Fig-1b, where the TEM foil was actually a layered "composite" of $\mathrm{Si}$ and $\mathrm{SiO} 2$ in the shallow trench isolation (STI), a rule of mixture was applied to estimate the mean free path. Compared to Scanning Capacitance Microscopy (SCM) and Atom Probe Tomography (APT), extending electron holography into 14nm FinFET on estimation of dopant profiles provides quick engineering feedbacks to wafer-foundries [3]. 

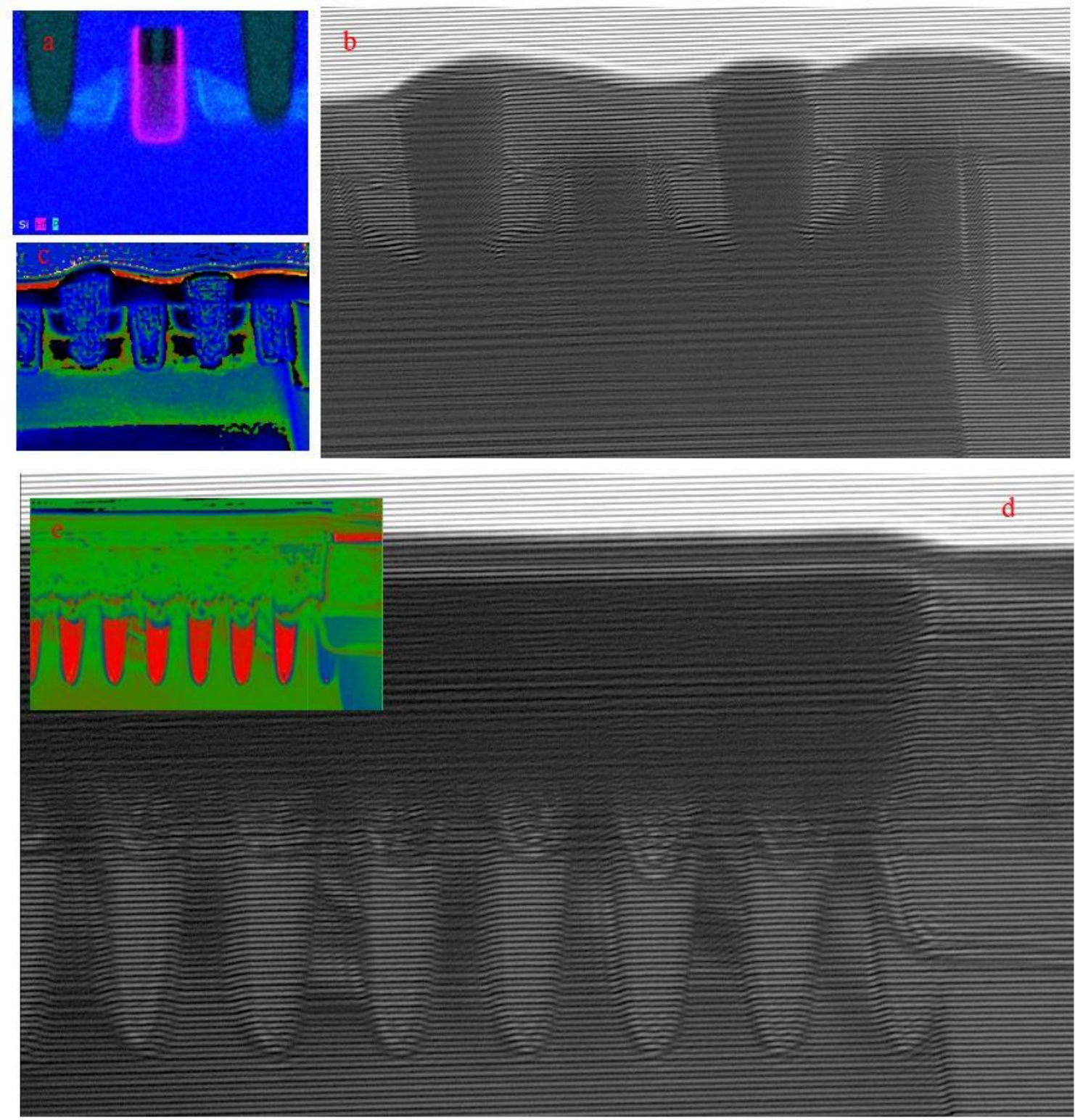

Figure 1. (a) X-ray EDS mapping for a long channel device from a TEM foil parallel to Fins; (b) BFelectron hologram from (a); (c) Inner Potential mapping extracted from (b); (d) BF electron hologram from a TEM foil parallel to Gate, and (e) Inner Potential mapping extracted from (d).

\section{References}

1. W. Zhao and Y. Wang, Microscopy \& Microanalysis, Vol. 21 (Supplement 1), (201), pp.1490 1491. 2. Y. Wang, et al., Ultramicroscopy, 124, (2013), pp. $117-129$.

3. Thanks to John Lemon and Frieder Baumann at Fab8 for inspiring discussions, and Management and Legal teams for supporting the publication clearance. 